\title{
Large extracellular vesicles in the left atrial appendage in patients with atrial fibrillation-the missing link?
}

\author{
Andreas Zietzer ${ }^{1,4}$ (1) $\cdot$ Baravan Al-Kassou ${ }^{1} \cdot$ Paul Jamme $^{1} \cdot$ Verena Rolfes $^{2} \cdot$ Eva Steffen $^{1} \cdot$ Marko Bulic $^{1}$. \\ Mohammed Rabiul Hosen ${ }^{1}$. Philip Roger Goody ${ }^{1}$. Vedat Tiyerili ${ }^{1}$. Sebastian Zimmer ${ }^{1}$. Jan Wilko Schrickel ${ }^{1}$. \\ Alexander Sedaghat ${ }^{1} \cdot$ Bernardo S. Franklin ${ }^{2} \cdot$ Nikos Werner $^{1,3} \cdot$ Georg Nickenig ${ }^{1} \cdot$ Felix Jansen $^{1,4}$
}

Received: 3 January 2021 / Accepted: 14 May 2021 / Published online: 1 June 2021

(c) The Author(s) 2021

\begin{abstract}
Atrial fibrillation (AF) is the most frequent arrhythmic disease in humans, which leads to thrombus formation in the left atrial appendage and stroke through peripheral embolization. Depending on their origin, large extracellular vesicles (lEVs) can exert pro-coagulant functions. In the present study, we investigated how different types of AF influence the levels of large EV subtypes in three distinct atrial localizations. Blood samples were collected from the right and left atrium and the left atrial appendage of 58 patients. $49 \%$ of the patients had permanent AF, $34 \%$ had non-permanent AF, and $17 \%$ had no history of AF. Flow cytometric analysis of the origin of the lEVs showed that the proportion of platelet-derived lEVs in the left atrial appendage was significantly higher in permanent AF patients compared to non-permanent AF. When we grouped patients according to their current heart rhythm, we also detected significantly higher levels of platelet-derived lEVs in the left atrial appendage (LAA) in patients with atrial fibrillation. In vitro studies revealed, that platelet activation with lipopolysaccharide (LPS) leads to higher levels of miR-222-3p and miR-223-3p in platelet-derived lEVs. Treatment with lEVs from LPS- or thrombin-activated platelets reduces the migration of endothelial cells in vitro. These results suggest that permanent atrial fibrillation is associated with increased levels of platelet-derived IEVs in the LAA, which are potentially involved in LAA thrombus formation.
\end{abstract}

Keywords Extracellular vesicle $\cdot$ Microvesicle $\cdot$ Platelet $\cdot$ Atrial fibrillation $\cdot$ microRNA-222-3p $\cdot$ microRNA-223-3p . Endothelial function

\section{Introduction}

Andreas Zietzer and Baravan Al-Kassou contributed equally.

Andreas Zietzer

andreas.zietzer@ukbonn.de

$\triangle$ Felix Jansen

felix.jansen@ukbonn.de

1 Medical Department II, University Hospital Bonn, Venusberg-Campus 1, 53127 Bonn, Germany

2 Institute of Innate Immunity, Medical Faculty, University of Bonn, Venusberg-Campus 1, 53127 Bonn, Germany

3 Medizinische Klinik III, Krankenhaus der Barmherzigen Brüder Trier, Nordallee 1, 54292 Trier, Germany

4 Medizinische Klinik und Poliklinik II, Innere Medizin, Kardiologie, Pneumologie und Angiologie, Universitätsklinikum Bonn, Venusberg-Campus 1, 53127 Bonn, Germany
Atrial fibrillation (AF) is the most frequent arrhythmic cardiac disease in humans and a significant health burden across the world [1,2]. AF is characterized by unorganized ectopic electrical activity in the atrium, which is caused by an atrial tissue substrate (typically local damage) [3]. While genetic susceptibility plays a certain role in the development of $\mathrm{AF}[4,5]$, it is primarily the remodeling of the atrial tissue that is both the cause and consequence of further arrhythmic episodes [6]. This inflicts a vicious circle of disease progression which starts with few, usually self-limiting, arrhythmic episodes (paroxysmal AF) but almost inevitably leads to permanent AF, if no treatment is administered [7]. The most serious consequence of $\mathrm{AF}$ is thrombus formation in the left atrium, which typically takes place in the left atrial appendage (LAA). Along with stasis of the blood in the LAA, impaired endothelial function has been shown to 
be an important trigger and a hallmark for atrial thrombus formation in atrial fibrillation [8-10]. Embolization of these thrombi into the cerebral arteries leads to stroke and makes $\mathrm{AF}$ the most important cause of embolic stroke [11, 12]. In this context, several studies have pointed out, that the risk of stroke in AF patients depends on the AF burden over time. This means that patients with permanent AF have a higher risk of stroke than patients with non-permanent $\operatorname{AF}[13,14]$. Recently, this concept has been challenged, because it is still not completely understood which factors are involved in atrial thrombus formation in AF [15].

In the context of thrombus formation, extracellular vesicles (EVs) and in particular platelet-derived EVs have been shown to be potent inductors of coagulation $[16,17]$. EVs are membranous bodies that are released by various cell types and serve as a means of intercellular communication $[18,19]$. One of the most significant determinants of the biological function of EVs is the levels of encapsulated microRNAs. These microRNAs can be transferred to and taken up by other cells and influence their biological behavior [20]. EVs are traditionally grouped into large EVs (formerly called microvesicles) and small EVs (formerly called exosomes). As large EVs can be analyzed directly by flow cytometry, we have focused on large EVs (lEV) for this study. A number of studies have found differences in the levels of EVs from different origins in the peripheral blood between non-AF and AF patients [21-24]. It has therefore been assumed that EVs are involved in the formation of atrial thrombi in AF patients. However, a specific analysis showing differences in thrombogenic EV levels in the left atrial appendage across different types of AF has not been performed.

In this study, we hypothesized that the subtype of AF (non-permanent vs. permanent) influences the level of procoagulatory platelet-derived lEVs in the LAA, which could serve as a path to investigate why thrombus formation and stroke are more common in permanent $\mathrm{AF}$ than in non-permanent AF patients.

\section{Material and methods}

\section{Patient recruitment}

For this analysis, 58 patients undergoing invasive procedures requiring venous access and transseptal puncture at the University Hospital Bonn were included. The recruitment took place from February 2018 until July 2019. Each patient underwent right and left atrial catherization for individual medical indications after written and informed consent had been obtained. Besides withdrawal of consent no further exclusion criteria were applied. The majority of the patients underwent mitral valve reconstruction, while few patients were treated with an occluder system of the LAA (Table 1). In all cases the catheter system was introduced via the right femoral vein. Directly after the transseptal puncture, unfractionated heparin was administered at a dose of 70-100 IE $/ \mathrm{kg}$ bodyweight to reach an activated clotting time of 250-300 s. For the definition of different types of AF, we used a simplified version of the 2016 ESC Guidelines [25]. In the first group, we included patients, who have never had AF. In the second group, we included patients with permanent $\mathrm{AF}$ without any reported attempt to restore a sinus rhythm for at least one year. In the third group, we included patients with intermittent episodes of AF but, who did not fulfill the criteria for permanent AF. In order to define coronary artery disease, the definition of the American heart association from 2013 was used: (at least one 50\% stenosis in the diameter of a major coronary artery) [26]. All the MitraClip patients underwent coronary angiography in preparation for the intervention. For the six remaining patients, the medical history was screened for coronary angiography reports. Echocardiographic routine measurements were performed in the facilities of the heart center at the University Hospital of Bonn. Aortic valve stenosis, mitral valve insufficiency and mitral valve stenosis were classified following recommendations of the European society of cardiology [27-29]. All echocardiographic data were reviewed by two independent cardiologists. The study was approved by the ethics committee of the University Hospital of Bonn (283/16) and is in accordance with the Declaration of Helsinki.

For the in vitro experiments, platelets were isolated from peripheral venous blood of healthy volunteers after written and informed consent had been obtained. The study was approved by the ethics committee of the University Hospital of Bonn (282/17) in accordance with the Declaration of Helsinki.

\section{Preparation of blood and isolation of EVs}

For the clinical analyses, all patients had fasted for at least $6 \mathrm{~h}$ before the catheter intervention. Blood was drawn through the catheter system from the right atrium (RA), the left atrium (LA), and the LAA. The blood was collected in trisodium citrate buffer to give a final citrate concentration of $3.2 \%$. Cells were immediately removed from the blood using a two-step centrifugation protocol with $1,500 \times g$ for $15 \mathrm{~min}$ and $13,000 \mathrm{rpm}$ for $2 \mathrm{~min}$, as previously reported $[30,31]$. The platelet-free plasma was then stored at $-80{ }^{\circ} \mathrm{C}$ until further analyses were performed. EVs were isolated by centrifugation at $20,000 \times g$ for 40 min with an additional washing step with PBS (Fig. 1). Further routine analyses, such as measurements of total cholesterol, HDL cholesterol, LDL cholesterol, triglycerides, lipoprotein a, creatinine, C-reactive protein, white blood cells, red blood cells, hemoglobin, and 


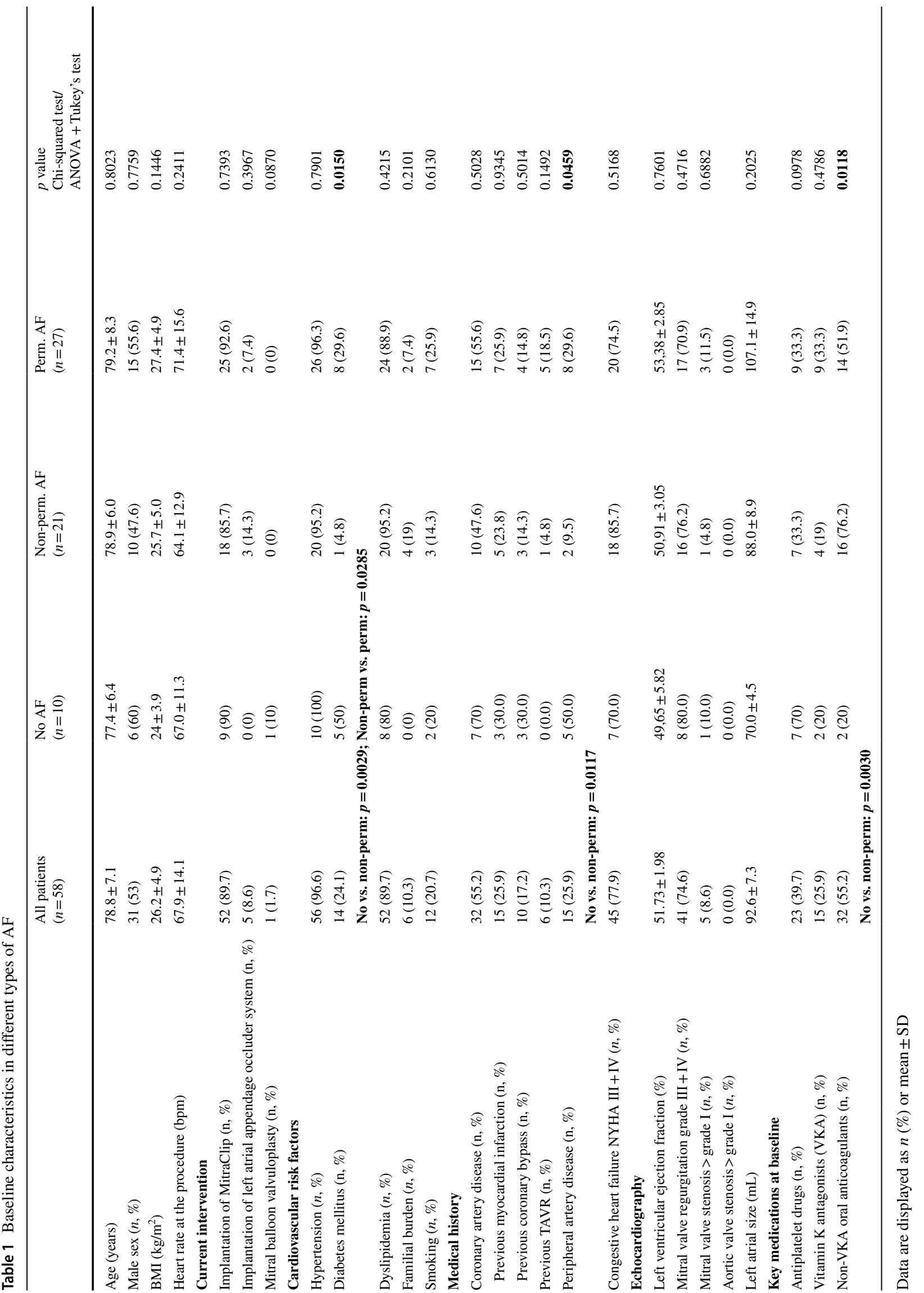


Fig. 1 a Diagram of lEV isolation procedure with representative FACS analysis at different steps of the isolation. b Electron microscopic imaging $1 \mathrm{EVs}$ by use of negative staining. $\mathbf{c}$ lEV size distribution as measured by nanoparticle tracking analysis
A Citrate plasma

$13000 \mathrm{rpm} \times 2 \mathrm{~min} 4^{\circ} \mathrm{C}$

Platelet free plasma<smiles>C=CCC1CCC1C=C</smiles>

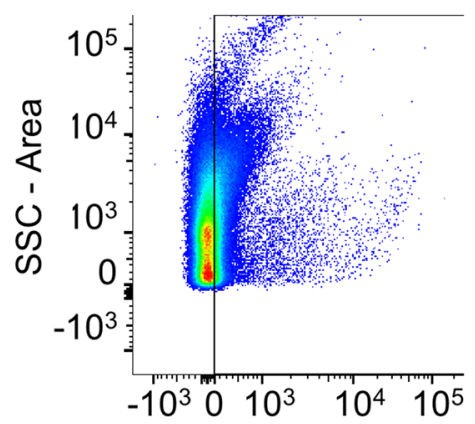

$2 \times 20,000 \mathrm{~g} \mathrm{x}$ $40 \min 4^{\circ} \mathrm{C}$<smiles>[CH]=C</smiles>

Remove supernatant<smiles>C1CC2C1C1CCC21</smiles><smiles>C=CC=C</smiles>

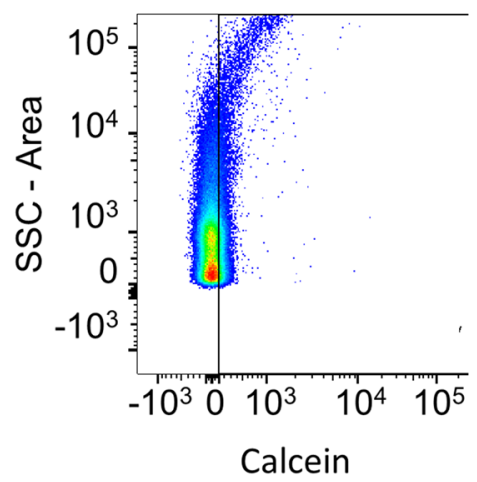

Large EVs for analysis

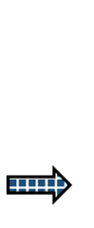

B

\section{Electron microscopic image of patient-derived IEVs}

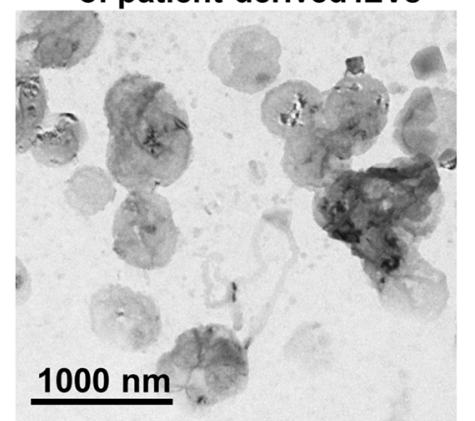

Size distribution of IEVs

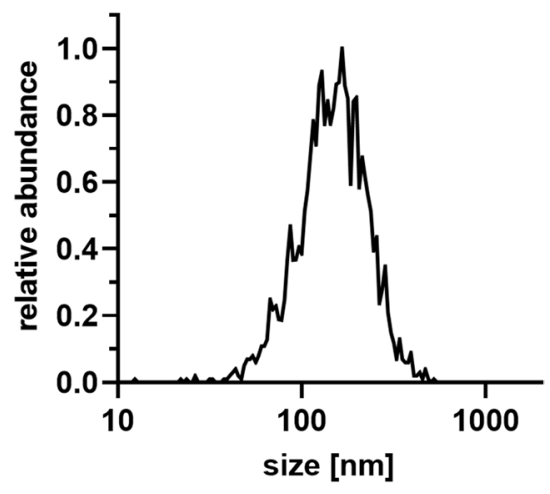


platelets were performed from additional venous blood samples in the central laboratory facilities of the Institute of Clinical Chemistry and Clinical Pharmacology at the University Hospital Bonn. Routine coagulation activity measurements including INR, aPTT, Factor II, Factor V, Factor VII, Factor VIII, and Factor X were performed at the facilities of the Institute for Experimental Hematology und Transfusion Medicine (IHT) at the University Hospital Bonn.

For the in vitro experiments, platelets were isolated from peripheral venous blood as previously reported [32]. In brief, other blood cells were removed by a twostep centrifugation at $330 \times g$ for $5 \mathrm{~min}$ and at $340 \times g$ for $10 \mathrm{~min}$. Prostaglandin E1 (PGE1; $200 \mathrm{nM}$ ) was used to inhibit platelet activation in the second step and for all subsequent centrifugation steps. Finally, the platelets were pelleted at $430 \times g$ for $15 \mathrm{~min}$. After counting in a hemocytometer, the platelets were resuspended in RPMI medium to achieve a concentration of $5 \times 10^{7}$ cells per ml.

\section{Flow cytometric analysis of IEV}

For flow cytometric analysis, the lEVs from $150 \mu \mathrm{l}$ of platelet-free plasma were used. The isolated IEVs were resuspended in $100 \mu \mathrm{PBS}$ and then incubated with $1 \mu \mathrm{l}$ anti-CD31-PE (BD Pharmingen, Cat\# 555446), $1 \mu \mathrm{l}$ antiCD41-APC (BD Pharmingen, Cat\# 559777), and $1 \mu \mathrm{l}$ anti-CD235-PE-Vio770 (Miltenyi Biotec, Cat\# 130-120614) antibodies for $45 \mathrm{~min}$ at RT. For specific staining of intact lEVs, $0.5 \mu \mathrm{L}$ of $1 \mathrm{mM}$ Calcein AM (Thermo Fisher Scientific, Cat\# C1430) in DMSO was added together with $400 \mu$ sterile PBS [33]. Following a $20 \mathrm{~min}$ incubation at RT, $50 \mu$ l of AccuCount Blank Particles 2.0-2.4 $\mu \mathrm{m}$ (Spherotech, Cat\# ACBP-20-10) were added for counting. A FACSCanto II (BD Bioscience) was used for sample analysis. Compensation, gating, and absolute quantification were performed with the software FlowJo V10 (BD Bioscience). To determine if the Calcein-stained particles are intact membranous bodies, $0.5 \mu \mathrm{L}$ Triton X-100 (Sigma-Aldrich, Cat\# T8787) were added to the sample after the last staining step to degrade the lEVs. This resulted in a Triton-X100 concentration close to $1 \%$ in the final sample. A swarming effect (detection of multiple EVs as one event) was excluded by use of a dilution series ranging from 50 to $250 \mu \mathrm{l}$ platelet-free plasma as the input for the analysis [34]. To assess the size of the plasma-derived EVs, Silica Microspheres, $1.0 \mu \mathrm{m}$ (Polysciences Cat\# 24326-15) and Silica Microspheres, $0.3 \mu \mathrm{m}$ (Polysciences Cat\# 24321-15) were used, as previously reported [35]. In brief, $0.5 \mu \mathrm{l}$ of the bead solutions were added to $200 \mu \mathrm{l}$ PBS and analyzed directly.

\section{Nanoparticle tracking analysis}

The size distribution of plasma-derived lEVs was assessed by nanoparticle tracking analysis in a ZetaView BASIC NTA-Nanoparticle Tracking Video Microscope PMX120 (Particle Metrix). To this end, EVs were isolated from $500 \mu \mathrm{L}$ plasma and finally resuspended in $500 \mu \mathrm{L}$ PBS. The samples were then further diluted 1:250 in PBS and analyzed. The concentration was confirmed to be in the linear range of the instrument.

\section{Electron microscopy of IEV}

Electron microscopic imaging was performed as previously reported [36]. In brief, IEVs isolated from $1 \mathrm{ml}$ plasma were finally resuspended in $10 \mu \mathrm{L}$ PBS with Protease Inhibitor Cocktail (Roche, Cat\# 4693132001). Prior to loading, the IEVs were diluted further 1:250 in PBS and $5 \mu$ of the suspension were loaded on Formvar-coated copper grids (Science Services, München). After incubation for $20 \mathrm{~min}$ at RT, the sample was then fixed for 5 min with $2 \%$ paraformaldehyde. Subsequently, the EVs were washed with PBS and fixed again for 5 min with $1 \%$ glutaraldehyde. Finally, the sample was contrast stained with $1.5 \%$ uranyl acetate for 4 min. Image acquisition was performed with a Jem2100 Plus (Jeol) operating at $200 \mathrm{kV}$ using a Gatan OneView $4 \mathrm{~K}$ camera.

\section{Platelet stimulation and IEV isolation}

The isolated platelets were stimulated with $200 \mathrm{ng} / \mathrm{ml}$ LPS or $1.0 \mathrm{U} / \mathrm{ml}$ thrombin or left untreated (native) for $3 \mathrm{~h}$. Subsequently, the platelets were removed from the suspension by centrifugation at $3000 \times g$ for $10 \mathrm{~min}$. The effective removal of platelets was confirmed by microscopic visualization in a hemocytometer. The supernatants were immediately frozen at $-80{ }^{\circ} \mathrm{C}$ until further use. For the isolation of in vitro-generated large platelet-derived EVs (abbreviated $\mathrm{pEV}$, to distinguish them from patient-derived platelet EVs), the supernatants were thawed on ice and $\mathrm{pEVs}$ were pelleted by centrifugation at $20,000 \times g$ for $40 \mathrm{~min}$, washed with PBS, and pelleted again.

\section{RNA isolation}

For the isolation of RNA from pEVs, pEVs isolated from $500 \mu \mathrm{L}$ of platelet supernatant were resuspended in 250 $\mu \mathrm{L}$ RNAse-free water before $750 \mu \mathrm{L}$ Trizol LS were added (Invitrogen, Cat\# 10296010). We used Caenorhabditis elegans miR-39 (cel-miR-39) RNA at a final concentration of $5 \mathrm{nmol} / \mathrm{L}$ (Qiagen) as a spike-in control, as previously reported [36]. To isolate RNA from the HCAEC samples, the cells were directly dissolved in Trizol (Invitrogen, Cat\# 
15596026) after washing with ice-cold PBS. RNA extraction with chloroform, isopropanol, and ethanol was conducted as previously reported [31]. The RNA was dissolved in pure $\mathrm{H}_{2} \mathrm{O}$. Purity $(260 / 280$ ratio $>1.8)$ and concentration of the isolated RNA were assessed with a NanoDrop2000 spectrophotometer (Thermo Fisher Scientific) as previously reported $[37,38]$.

\section{microRNA quantification with TaqMan assays by qPCR}

10 ng of total RNA were used for cDNA preparation with the TaqMan microRNA Reverse Transcription kit (Applied Biosystems, Cat\# 4366596), adhering to the manufacturer's protocols. $1 \mu \mathrm{L}$ of the resulting cDNA solution was used for quantitative real-time PCR with the respective TaqMan probe (hsa-miR-126-3p Cat\# 4427975, Assay ID 002228; hsa-miR-222-3p, Cat\# 4427975, Assay ID 000525; hsa-miR223-3p, Cat\# 4427975, Assay ID 000526; cel-miR-39-3p, Cat\# 4427975, Assay ID 000200; RNU6b, Cat\# 4427975, Assay ID 001093) and TaqMan Universal Master Mix II (Applied Biosystems, Cat\# 4440040) in a 7500 HT RealTime PCR instrument (Applied Biosystems). MiR levels in pEVs were calculated as $2^{-\mathrm{ddCT}}$ vs. the spike-in control cel-miR-39. For the experiments with HCAECs, cellular miR-expression was calculated as $2^{-\mathrm{ddCT}}$ vs RNU6b as an internal control.

\section{Scratch-wound assay}

To assess the effect of pEVs on EV recipient cells, we used endothelial cells, because endothelial cells are the only resident cells in the LAA that are in direct contact with bloodderived EVs. As endocardial endothelial cells from the LAA are not commercially available, we used female primary human coronary artery endothelial cells (HCAECs) from passage 8 (Promocell, Cat\# C-1222) for basic migration experiments in a scratch-wound assay. The assay was performed as previously described [39]. In brief, HCAECs were allowed to grow to confluency in a 6-well plate. The pEVs from $500 \mu \mathrm{l}$ of supernatant were resuspended in $500 \mu \mathrm{l}$ fresh RPMI medium and further diluted 1:1 in Endothelial Cell Growth Medium MV with supplements (Promocell, Cat\# C-22020) before incubation with the HCAECs. Directly after stimulation, a scratch was applied to the center of the well with a sterile $200 \mu \mathrm{l}$ pipet tip. The scratch was photographed in a marked position at $0,2,4$, and $6 \mathrm{~h}$. To calculate the migratory activity of the cells, the remaining cell-free area was measured and compared to the cell-free area at $0 \mathrm{~h}$. Image acquisition and analyses were conducted with a Zeiss Axio Observer microscope and the ZEN 2.3 pro software.

\section{Transfection of miRs into HCAECs}

To simulate an overexpression of miR-222-3p and miR223-3p in HCAECs, we used miR-222-3p-mimic, miR-2233p-mimic, or control RNA (all Invitrogen: miR-222-3p mirVana miRNA mimic, Cat\# 4464066, Assay ID MC11376; mir-223-mirVana miRNA mimic, Cat\# 4464084, Assay ID MC24077, mirVana miRNA mimic Negative Control 1, Cat\# 4464060). The RNAs were transfected at a final concentration of $10 \mathrm{nmol} / \mathrm{L}$ by use of Lipofectamine RNAiMAX Transfection reagent (Invitrogen, Cat\# 13778150) within $24 \mathrm{~h}$ of incubation. Effective overexpression of the miRs was confirmed via qPCR. The Wound-scratch assays were started $24 \mathrm{~h}$ after the transfection.

\section{Statistical analysis}

Statistical analyses were performed using the software Prism8 (GraphPad). The 1EV data in Fig. 3 were analyzed by a two-way ANOVA followed by Tukeys's multiple comparison test. The distribution of the data in Fig. 4 differed significantly from a normal distribution as assessed by the D'Agostino and Pearson's test. Therefore, a Mann-Whitney $U$ test was used to analyze the data in Fig. 4 . For the in vitro experiments, means of two groups were compared with an unpaired t-test and means of more than two groups were compared by a one-way ANOVA followed by Tukeys's multiple comparison test. Categorial variables in the baseline characteristics were compared with the Chi-squared test. The number of independent experiments as well as the applied tests for statistical significance are reported in the figure legends. All reported $p$ values are two-sided. The data presented in this study are available from the corresponding author upon request.

\section{Results}

For the flow cytometric analysis of blood-derived lEVs, we included 58 patients with a mean age of $78.8 \pm 7.1$ years, equally distributed between both sexes ( $47 \%$ female $53 \%$ male) and with a mean body mass index of $26.2 \pm 4.9 \mathrm{~kg} / \mathrm{m}^{2}$ (Table 1). The patients were divided into three groups: (i) patients without any history of AF, (ii) patients with nonpermanent AF, and (iii) patients with permanent AF [25]. The heart rate of the patients did not differ significantly at the time of the sample collection between the groups of AF. No differences between these groups were detected for cardiovascular risk factors, with the exclusion of diabetes mellitus, which showed a significantly higher prevalence in the no AF group compared to the other two groups. When considering the patients' medical history, the prevalence of peripheral artery disease was significantly higher in the 
no AF group compared to the permanent AF group. No differences were detected for the prevalence of coronary artery disease, previous myocardial infarction, coronary bypass, transfemoral aortic valve replacement, congestive heart failure (NYHA III + IV). Routine echocardiographic imaging revealed a tendency towards larger LA volumes in patients with permanent AF (Table 1). The differences were not statistically different. No differences were found for mitral valve regurgitation (grade III + IV), mitral valve stenosis (grade $>$ I) or left ventricular ejection fraction. No cases of aortic valve stenosis (grade $>$ I) were detected in our collective. Also, when considering the drugs the patients had been taking that have a direct effect on coagulation and platelet activation, patients in the non-permanent AF group more frequently received non-VKA anticoagulants, while there was no difference for anti-platelet drugs. Routine laboratory parameters at baseline showed no differences for parameters of cardiovascular risk, inflammation, or kidney function (Table 2). However, the coagulation factors II, V, VII, and X were significantly decreased in the groups with $\mathrm{AF}$, which was expected due to their higher rate of treatment with anticoagulants.

lEVs were isolated from citrate plasma from the three different atrial locations (described in the Methods section) of the patients and characterized by electron microscopy, nanoparticle tracking analysis, and flow cytometry. We found a size distribution ranging from 80 to $800 \mathrm{~nm}$, with a peak around $200 \mathrm{~nm}$ (Figs. 1B, C, 2A). The specific staining of intact $1 E V$ s by Calcein AM was assessed in a lysis experiment, where $1 \%$ Triton- $X$ was added just before analysis in the cytometer. After lysis with Triton-X, the IEV signal disappeared completely (Fig. 2B). To exclude a swarming effect, which is characterized by the detection of multiple lEVs as one event, we performed a dilution series by varying the amount of $1 E V$ input into the analysis. Increasing or decreasing the number of $1 \mathrm{EV}$ input led to directly proportional changes in the detection of events, which suggests that swarming is not a factor (Figure S1). To further distinguish between different cellular EV subtypes, we applied the following gates: platelet-derived EVs $\left(\right.$ Calcein $^{+} /$ $\left.\mathrm{CD} 41^{+} / \mathrm{CD} 31^{+}\right)$, endothelial cell-derived EVs $\left(\right.$Calcein $^{+} /$ CD $41^{-} / \mathrm{CD} 31^{+}$) (Fig. 2C), and red blood cell-derived EVs $\left(\right.$ Calcein $^{+} / \mathrm{CD} 235 \mathrm{a}^{+}$) (Fig. 2D).

Quantification of the total number of EVs revealed no significant difference between the three groups of patients across the three atrial locations (Fig. 3A). With regard to the different EV subtypes, we found that exclusively in the LAA the relative portion of platelet-derived EVs was
Table 2 Key laboratory parameters at time of inclusion in the study

\begin{tabular}{|c|c|c|c|c|c|}
\hline & $\begin{array}{l}\text { All patients } \\
(n=58)\end{array}$ & $\begin{array}{l}\text { No AF } \\
(n=10)\end{array}$ & $\begin{array}{l}\text { Non-perm. AF } \\
(n=21)\end{array}$ & $\begin{array}{l}\text { Perm. AF } \\
(n=27)\end{array}$ & $\begin{array}{l}p \text { value } \\
\text { ANOVA + Tuk- } \\
\text { ey's test }\end{array}$ \\
\hline Total cholesterol [mg/dl] & $154.9 \pm 45.8$ & $158.6 \pm 49.3$ & $158.5 \pm 39.3$ & $150.8 \pm 50.4$ & 0.8286 \\
\hline HDL-chol. [mg/dl] & $50 \pm 13.7$ & $50.6 \pm 14.3$ & $53.1 \pm 11.2$ & $47.3 \pm 15.1$ & 0.3427 \\
\hline LDL-chol. [mg/dl] & $94 \pm 38.7$ & $94.5 \pm 36.8$ & $88.9 \pm 32.1$ & $97.8 \pm 44.5$ & 0.7392 \\
\hline Triglycerides [mg/dl] & $99.0 \pm 52.8$ & $96.7 \pm 61.8$ & $110.8 \pm 55.3$ & $90.9 \pm 47.8$ & 0.4653 \\
\hline Lipoprotein a [nmol/1] & $61.6 \pm 104.8$ & $89.2 \pm 66.9$ & $79.3 \pm 159.4$ & $35.4 \pm 50.9$ & 0.3635 \\
\hline Creatinine $[\mathrm{mg} / \mathrm{dl}]$ & $1.55 \pm 1.04$ & $1.87 \pm 1.14$ & $1.35 \pm 0.58$ & $1.59 \pm 1.26$ & 60.4201 \\
\hline C-reactive protein $[\mathrm{mg} / \mathrm{l}]$ & $15.91 \pm 24.31$ & $21.46 \pm 31.09$ & $16.77 \pm 27.3$ & $3 \quad 13.07 \pm 19.20$ & 00.6512 \\
\hline INR [] & $1.3 \pm 0.4$ & $1.2 \pm 0.3$ & $1.2 \pm 0.3$ & $1.5 \pm 0.5$ & 0.0544 \\
\hline aPTT $[\mathrm{s}]$ & $29.2 \pm 5.3$ & $27.4 \pm 3.4$ & $29.1 \pm 6.3$ & $30 \pm 4.9$ & 0.4276 \\
\hline White blood cells $\left[10^{9} / 1\right]$ & $7.1 \pm 2.3$ & $6.7 \pm 2.3$ & $7.7 \pm 2.7$ & $6.9 \pm 1.8$ & 0.3866 \\
\hline Red blood cells $\left[10^{12} / 1\right]$ & $3.7 \pm 0.7$ & $3.8 \pm 0.8$ & $3.7 \pm 0.7$ & $3.7 \pm 0.5$ & 0.7942 \\
\hline Hemoglobin [g/dl] & $11.1 \pm 2.2$ & $11 \pm 2.3$ & $11.3 \pm 2.4$ & $11.0 \pm 2.1$ & 0.8917 \\
\hline Platelets $\left[10^{9} / 1\right]$ & $208 \pm 64.2$ & $200 \pm 65.2$ & $218 \pm 72.3$ & $202.4 \pm 57.2$ & 0.6658 \\
\hline \multirow[t]{2}{*}{ Factor II [\%] } & $75.1 \pm 25.8$ & $84.1 \pm 17.8$ & $83.2 \pm 26.4$ & $63.8 \pm 24.7$ & 0.0183 \\
\hline & \multicolumn{5}{|c|}{ Non-perm. vs. perm. $p=0.0289$} \\
\hline \multirow[t]{2}{*}{ Factor V $[\%]$} & $93.5 \pm 24.4$ & $109.3 \pm 33.4$ & $96.2 \pm 22.8$ & $84.1 \pm 17$ & 0.0166 \\
\hline & \multicolumn{5}{|c|}{ No vs. perm. $p=0.0150$} \\
\hline \multirow[t]{2}{*}{ Factor VII [\%] } & $71.2 \pm 31$ & $84.4 \pm 33.6$ & $81.4 \pm 29$ & $56.2 \pm 26$ & 0.0067 \\
\hline & \multicolumn{5}{|c|}{ No vs. perm. $p=0.0322$; Non-perm. vs. perm. $p=0.0145$} \\
\hline Factor VIII [\%] & $202.2 \pm 60.6$ & $227.6 \pm 62.6$ & $203.8 \pm 60.5$ & $189.7 \pm 58.7$ & 0.2560 \\
\hline \multirow[t]{2}{*}{ Factor X [\%] } & $72 \pm 31.2$ & $78.9 \pm 27.4$ & $81.9 \pm 29.8$ & $59.9 \pm 31.1$ & 0.0448 \\
\hline & \multicolumn{5}{|c|}{ Non-perm. vs. perm. $p=0.0472$} \\
\hline
\end{tabular}

Data are displayed as $n(\%)$ or mean $\pm \mathrm{SD}$ 
A

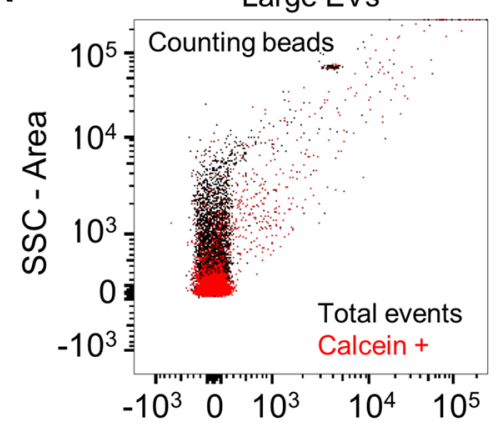

B Triton- $X$ degradation of IEVs

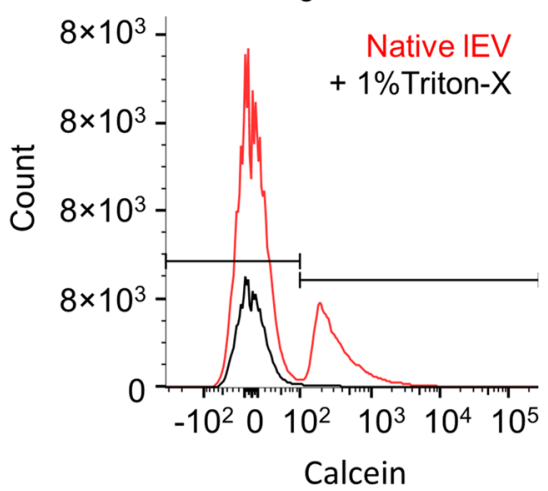

C

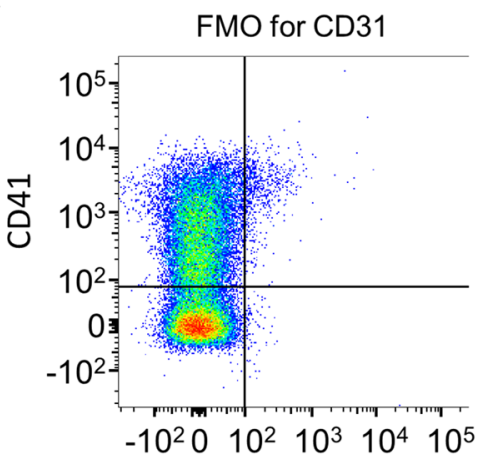

D

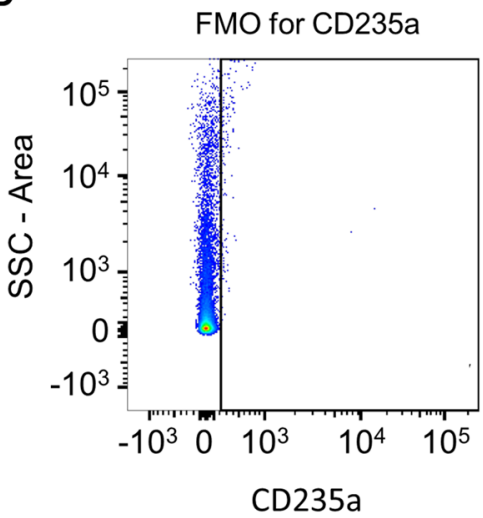

Silica beads

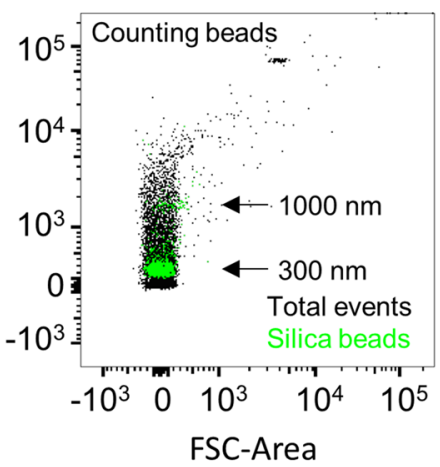

FSC-Area

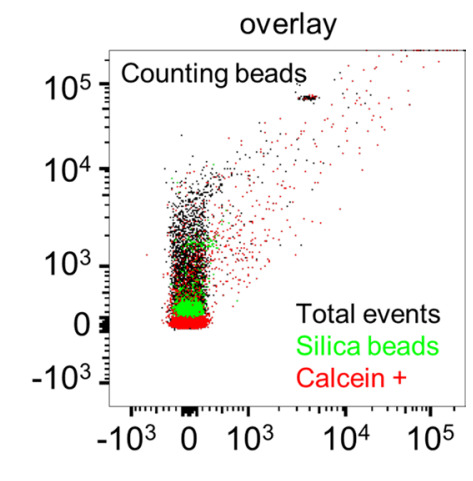

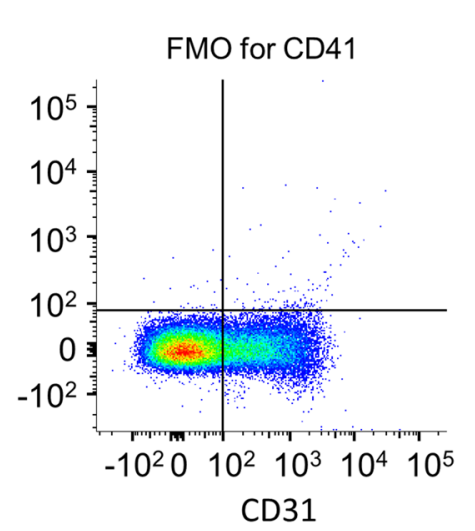

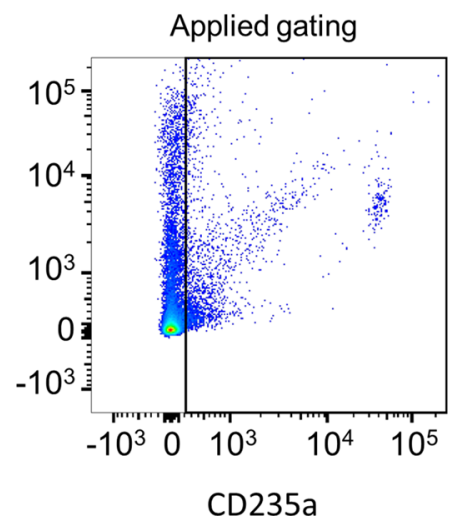

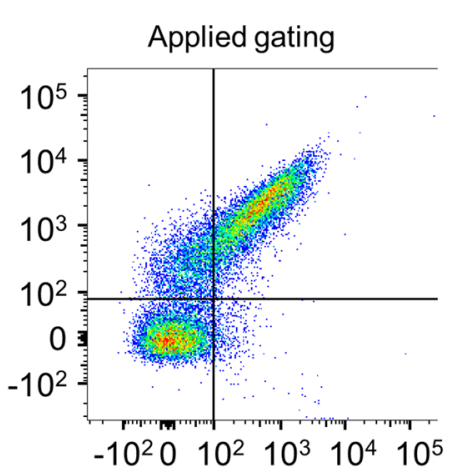


4Fig. 2 a Silica beads that have a 300 or $1000 \mathrm{~nm}$ diameter and a comparable refractory index as $1 \mathrm{EVs}$ were used to estimate the size distribution of the lEVs by flow cytometry. $\mathbf{b}$ Effective staining of intact lEVs was confirmed by a lysis experiment with $1 \%$ Triton-X 100 . c, d Gating strategy for the flow cytometric analyses of different $1 \mathrm{EV}$ subtypes. Calcein AM-positive $\left(\right.$ Calcein $\left.^{+}\right)$events were identified as displayed in Fig. 1. Further gates were established by fluorescenceminus-one controls (FMO) for CD41-APC, CD31-PE, and CD235aPE-Vio770. Platelet-derived EVs $\left(\mathrm{Calcein}^{+} / \mathrm{CD} 41^{+} / \mathrm{CD} 31^{+}\right)$, endothelial cell-derived EVs $\left(\right.$ Calcein $\left.{ }^{+} / \mathrm{CD} 41^{-} / \mathrm{CD} 31^{+}\right)$, and red blood cell-derived EVs $\left(\mathrm{Calcein}^{+} / \mathrm{CD} 235 \mathrm{a}^{+}\right)$were gated as illustrated

significantly higher in permanent AF patients compared to non-permanent AF. No changes were detected for endothelial cell-derived EVs or red blood cell-derived EVs across the three patient groups and locations (Fig. 3B).

To confirm the finding that AF influences the distribution of $1 \mathrm{EV}$ subtypes in the LAA, we regrouped our patients by current heart rhythm at the time of the blood collection (sinus rhythm vs. AF). Again, no significant differences were detected in total $1 \mathrm{EV}$ numbers, but the relative portion of platelet-derived $1 E V$ s was significantly higher in the LAA and slightly lower in the RA in the AF group (Fig. 4A). Endothelial cell-derived $1 E V$ s were significantly lower in the RA of AF patients, whereas no difference was found for endothelial cell-derived $1 E V s$ in the LAA between the sinusrhythm and AF groups (Fig. 4B). Red blood cell-derived lEVs were not significantly different between patients in sinus rhythm compared to AF patients for any of the atrial locations studied.

Interestingly, only platelet-derived $\mathrm{IEV}$ levels in the LAA were increased in AF patients (Fig. 3B), while total platelet levels were unchanged (Table 2). Therefore, we inferred that $\mathrm{AF}$ is associated with platelet activation, leading to increased levels of platelet-derived IEVs in the LAA, as previously reported [40].

As a next step, we wanted to further characterize the biological function of in vitro-generated, large platelet-EVs (pEVs) upon activation. To this end, platelets were isolated from healthy donors and used to generate $\mathrm{pEVs}$ in vitro, with and without prior stimulation with LPS or thrombin. In the pEVs, we measured the levels of three functionally important and highly abundant microRNAs by qPCR: miR-126-3p [41], miR-222-3p [42], miR-223-3p [40, 43]. We found that vesicular miR-222-3p and miR-223-3p levels were significantly increased after stimulation with LPS, while activation with thrombin only lead to less-pronounced and, therefore, non-significant changes. The levels of miR-126-3p in the $\mathrm{pEVs}$ remained unchanged across the conditions (Fig. 5A). To test the impact of LPS and thrombin activation on $\mathrm{pEV}$ function, we incubated the pEVs with otherwise native HCAECs and performed a migration assay. Endothelial regeneration is an important factor involved in left atrial thrombus formation and thus particularly relevant in this context [9]. We found that both, LPS and thrombin activation of platelets, induced the release of $\mathrm{pEVs}$, which significantly inhibited endothelial cell migration. No differences were detected upon incubation with native $\mathrm{pEVs}$ or with supernatants depleted of pEVs. These findings were mirrored by increased levels of miR-222-3p and miR-223 in the recipient HCAECs after treatment with $\mathrm{pEVs}$ from thrombin- and LPS-activated platelets, respectively. Endothelial miR-126-3p levels remained unchanged by $\mathrm{pEV}$ treatment.

The anti-angiogenic potential of miR-222-3p and miR223-3p was confirmed in a transfection experiment. We found HCAEC migration to be significantly reduced after experimental overexpression of miR-222-3p as well as miR223-3p (Fig. 6A, B).

\section{Discussion}

In this study, we show for the first time, that the type of atrial fibrillation present in a patient has an effect on the distribution of $1 \mathrm{EV}$ subtypes in the left atrial appendage. In vitro, we found that EVs from activated platelets exhibit significantly higher levels of miR-222-3p and miR-223-3p and reduce the migratory potential of the endothelial EV-recipient cells.

Previous studies have shown, that the level of plateletderived lEVs is significantly higher in the peripheral venous blood of non-valvular AF patients, compared to patients who have no history of AF [21]. Similar results were obtained by Azzam and co-workers, who found increased levels of platelet-derived EVs in patients with valvular AF [22]. Ederhy and co-workers found that, besides higher levels of plateletderived EVs, also endothelial-cell-derived EVs were higher in patients with AF compared to healthy patients. These differences collapsed, however, when AF patients were compared to a patient collective with a similar cardiovascular risk profile but without AF [22]. In our study, we did not analyze peripheral venous blood, but instead we found elevated levels of platelet-derived EVs in blood from the RA of AF patients, which is the closest location to peripheral veins that we tested. In our study, endothelial-cell-derived EVs were lower in the RA of AF patients. When comparing the baseline characteristics of the groups in our study, no significant differences were detected for most cardiovascular risk factors, age, or other confounding factors, such as kidney disease, anemia, heart failure, coronary artery disease, and valvular defects (Tables 1,2). Only the prevalence of diabetes was higher in the control group, which is unlikely to explain why EV levels in the AF group were higher [44].

A very recent study also analyzed $\mathrm{EV}$ in the left atrial appendage in AF patients [24]. However, different from our analysis, the blood for extracting the EVs was taken during open heart surgery, a procedure that is very invasive and may itself cause activation of various blood cells. Furthermore, 

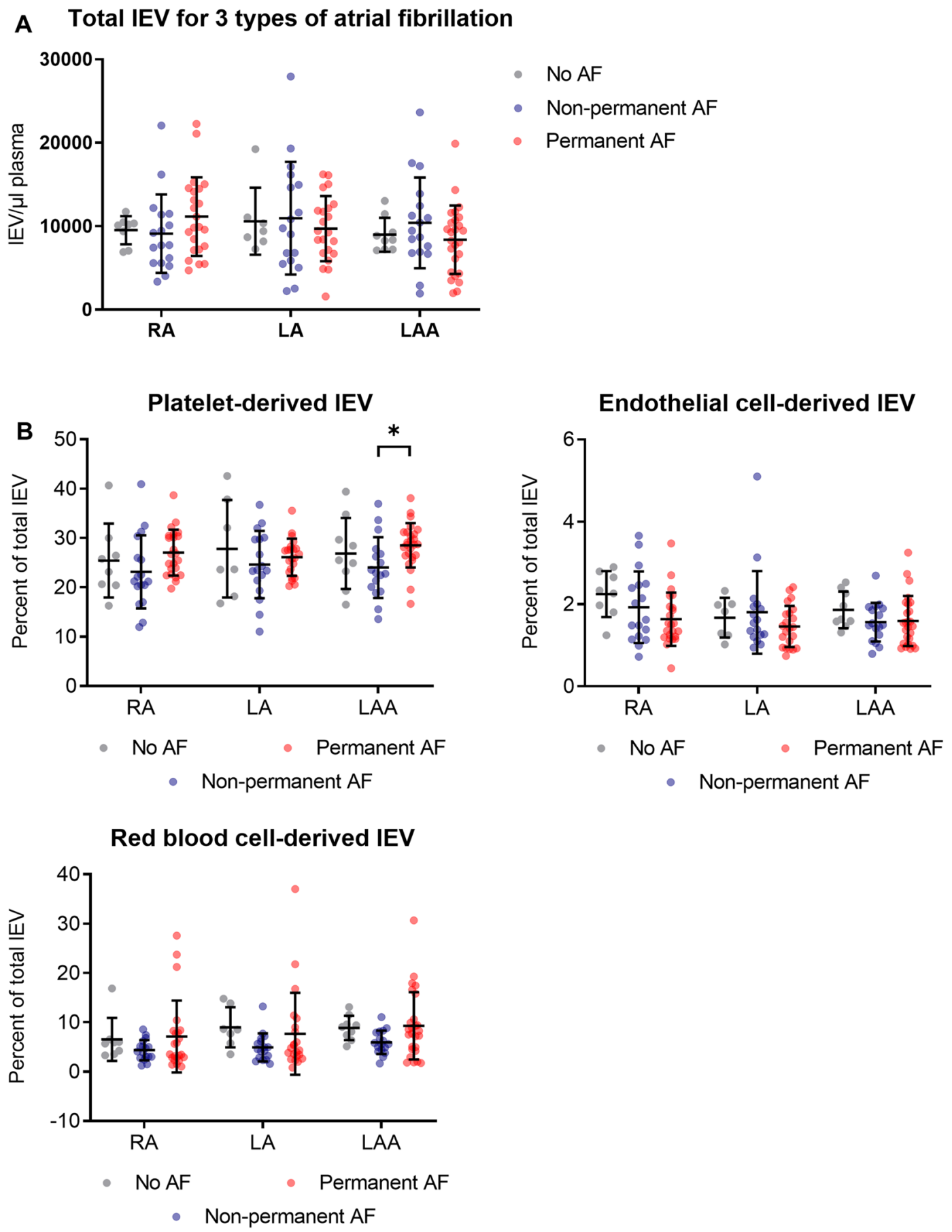

Fig. 3 Flow cytometric analysis of $1 E V s$ in three atrial locations $(R A$ right atrium, $L A$ left atrium, $L A A$ left atrial appendage) grouped by different types of atrial fibrillation (no history of AF, non-permanent $\mathrm{AF}$, permanent $\mathrm{AF}$ ). a Total $1 \mathrm{EV}$ numbers per location $\left(\right.$ Calcein $^{+}$ events) b Percentage of platelet-derived 1EVs, endothelial cell-

derived $\mathrm{IEVs}$, and red blood cell-derived EVs at the three atrial locations in different types of atrial fibrillation. $n=10 / 21 / 27$. Data are presented as individuals with the mean $\pm \mathrm{SD} ; * p<0.05$; two-way ANOVA + Tukey's multiple comparison test

the authors used an EV array to distinguish between the EV single particle basis. Interestingly, it was found that tissue subtypes, which does not allow for the analysis of EVs on a factor bearing EVs, were significantly increased in the LAA 
A

Total IEV in patients with and without current AF

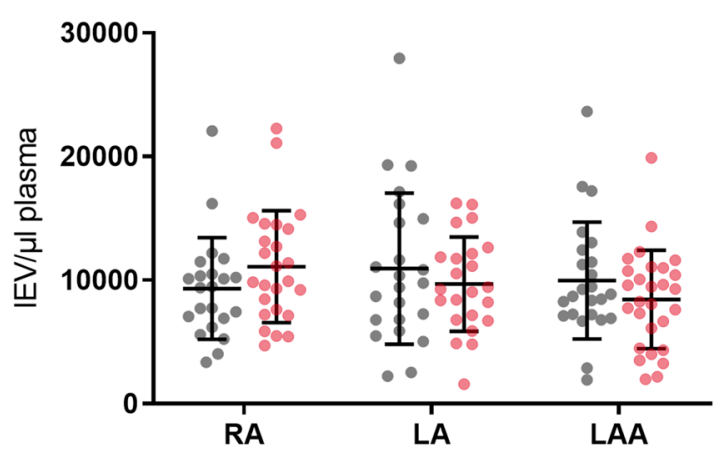

- Sinus rhythm

- Atrial fibrillation

B

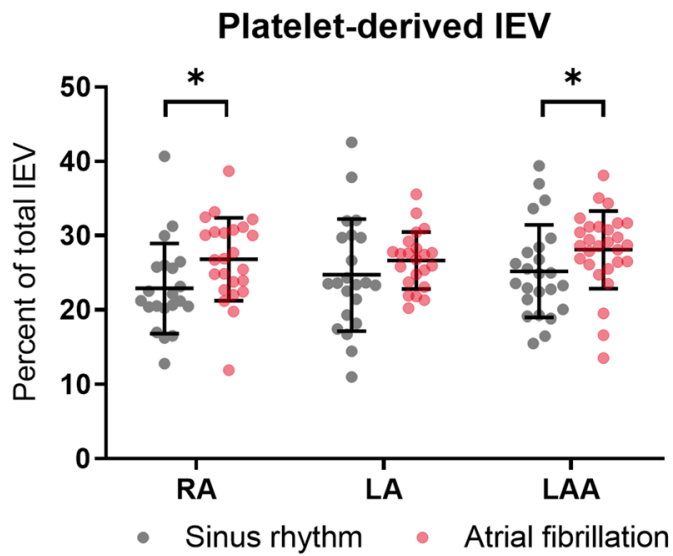

Red blood cell-derived IEV

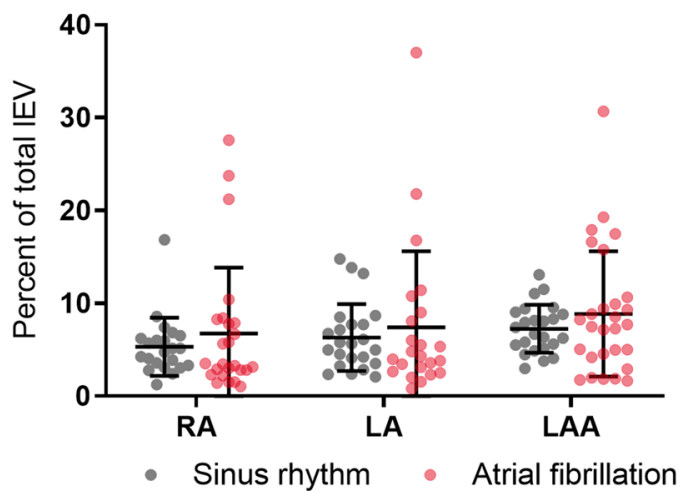

Fig. 4 Flow cytometric analysis of $1 \mathrm{EVs}$ in three atrial locations (RA, LA, LAA) grouped by current heart rhythm at the time of catheterization (sinus rhythm vs. atrial fibrillation). a Total lEV numbers per location $\left(\right.$ Calcein ${ }^{+}$events) b Percentage of platelet-derived lEVs,

in AF patients. Tissue factor bearing EVs had previously shown to be involved in thrombosis and to be particularly relevant in the context of malignant diseases [45-47].

In another very interesting study, Pourtau and co-workers found a decrease in the pro-coagulatory activity of EVs in

\section{Endothelial cell-derived IEV}

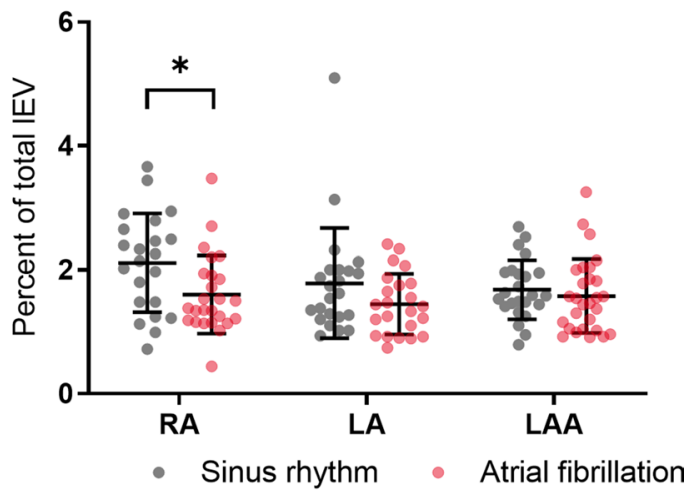

endothelial cell-derived $1 E V s$, and red blood cell-derived $1 E V s$ at the three atrial locations grouped by current heart rhythm. $n=10 / 21 / 27$. Data are presented as individuals with the mean $\pm \mathrm{SD} ; * p<0.05$, Mann-Whitney $U$ test

the LA after induction of AF [23]. These results were attributed to increased consumption of the EVs during AF by coagulation, however, no measurements of EV levels were performed in the left atrium. 
A miR-126-3p
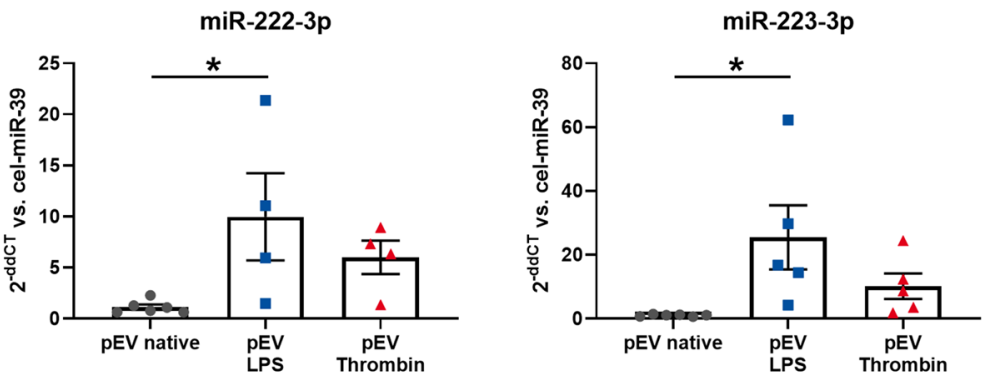

B

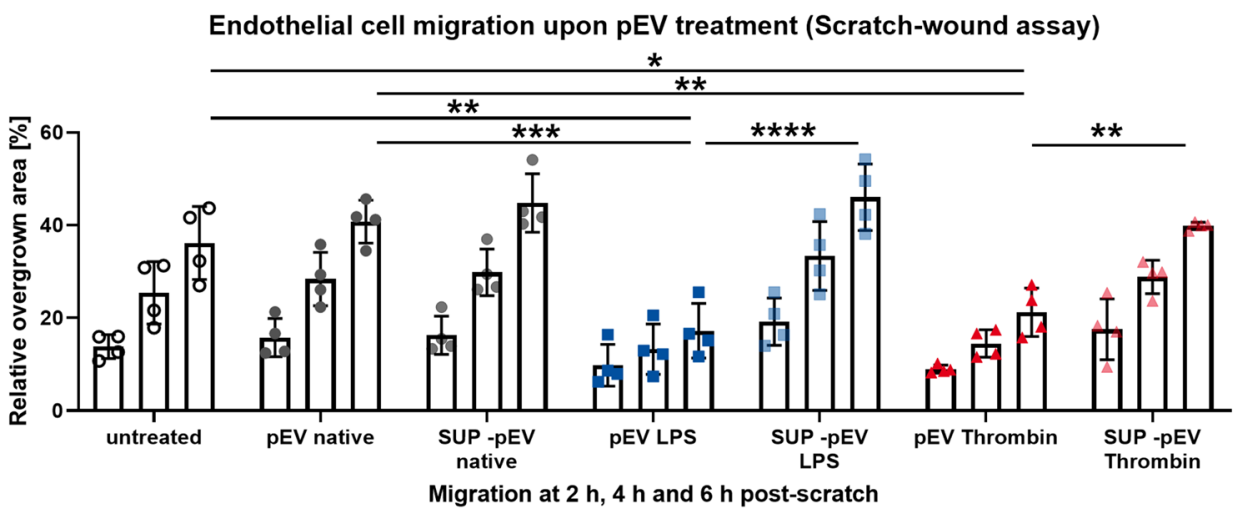

C
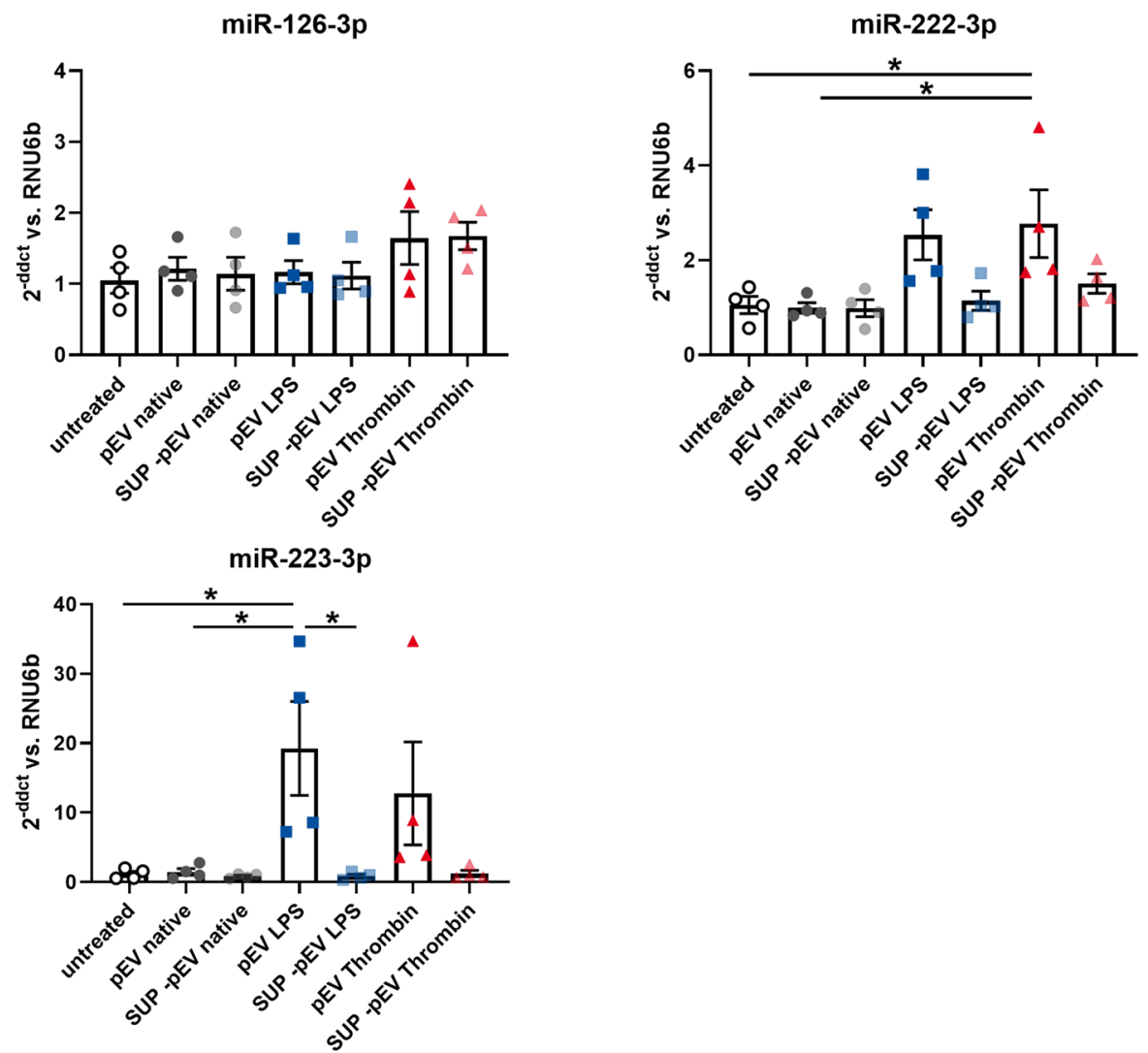

From the data in our study, we can exclude that the higher proportion of platelet-derived EVs in the LAA are caused by higher levels of platelets in the AF patients, because no differences in platelet levels were detected between the groups. Therefore, it seems plausible, that the differences are the result of increased platelet activation 
4Fig. 5 a Quantification of miR-126-3p, miR-222-3p, and miR-223-3p in pEVs derived from native platelets and after stimulation with LPS or thrombin by qPCR. miR levels are expressed as $2^{-\mathrm{ddCT}}$ vs. celmiR-39, which was added as a spike-in reference, $n=4-6$. b Endothelial cell migration was assessed in a scratch-wound assay after stimulation with the respective pEVs or pEV-depleted supernatants, $n=4$. c Quantification of miR-126-3p, miR-222-3p, and miR-223-3p in HCAECs after stimulation with pEVs or pEV-depleted supernatants. miR levels are expressed as $2^{-\mathrm{ddCT}}$ vs RNU6b, $n=4$. Data are presented as individuals with the mean $\pm \mathrm{SEM} * p<0.05$, $* * p<0.01$, $* * * p<0.001, * * * * p<0.0001$, ANOVA + Tukey's multiple comparison test

and EV release in the LAA of AF patients. This would be in line with previous reports of platelet activation leading to the release of EVs [40].

The effect of platelet activation on platelet-derived EV function was further investigated in vitro. Endothelial-cell function and endothelial healing have been implicated in atrial thrombus formation $[9,10]$. Therefore, we were interested in three miRs, which are known to strongly influence endothelial-cell function: miR-126-3p is known to promote endothelial regeneration [48], and miR222-3p and miR-223-3p inhibit endothelial regeneration

A

Endothelial cell migration after miR-222-3p ransfection (Scratch-wound assay)

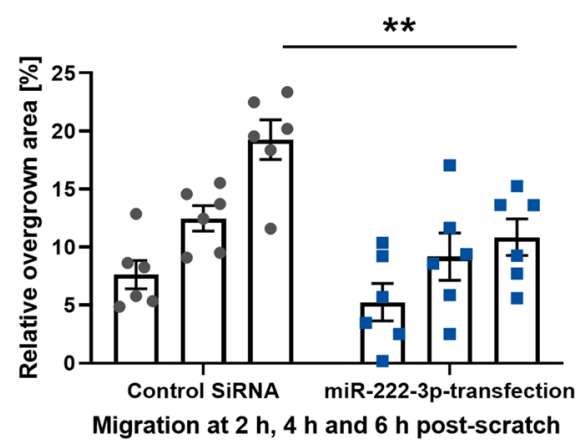

B

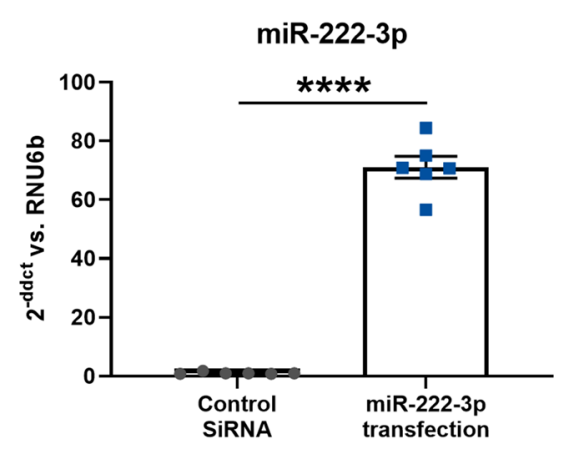

Fig. 6 a Endothelial cell migration was assessed in a scratchwound assay after transfection of miR-222-3p and miR-223-3p, $n=6$. b Quantification of miR-222-3p, and miR-223-3p in HCAECs after transfection of miR-222-3p and miR-223-3p. miR levels are
$[49,50]$. After platelet activation, the released EVs contained significantly higher levels of the anti-regenerative miR-222-3p and miR-223-3p. The EVs were loaded with anti-angiogenic miR-222-3p and miR-223-3p, which then caused the regenerative capacity to be lower in endothelial EV-recipient cells. This endothelial phenotype after EV stimulation was accompanied by increased mir-222-3p and miR-222-3p levels in the endothelial EV-recipient cells. These results confirm the previous findings that platelet activation can increase vesicular miR-223-3p transfer to endothelial recipient cells in a functionally relevant manner [51].

Unfortunately, our study also has some limitations. The number of the patients we included in our study is higher than in most other investigations of $1 \mathrm{EV}$ levels in $\mathrm{AF}$ patients, but still some differences in the EV levels of less abundant EVs, such as endothelial-cell-derived EVs, might not have been detected due to insufficient power.

In summary, we provide for the first evidence that the relative amount of platelet-derived EVs is higher in the left atrial appendage in patients with permanent AF, compared to patients with non-permanent AF. This may lead to
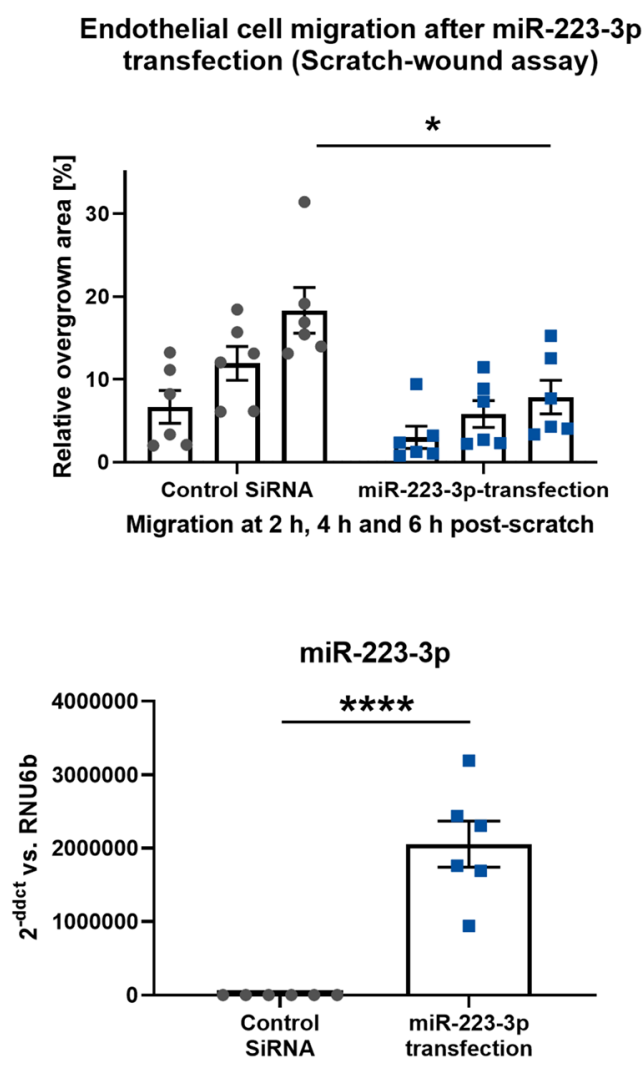

expressed as $2^{-\mathrm{ddCT}}$ vs RNU6b, $n=6$. Data are presented as individuals with the mean \pm SEM; ${ }^{*} p<0.05,{ }^{* *} p<0.01, * * * * p<0.0001$, unpaired $t$ test 
increased transfer of miR-223-3p into endothelial cells and consequently to reduced endothelial healing, which has been shown to be thrombogenic itself. This work can therefore serve as a basis for further investigations of the role of EVs in thrombus formation, specifically in the milieu of the left atrial appendage.

Supplementary Information The online version contains supplementary material available at https://doi.org/10.1007/s00392-021-01873-4.

Acknowledgements We would like to acknowledge the assistance of the Flow Cytometry Core Facility at the Institute of Experimental Immunology, especially from Andreas Dolf, Medical Faculty at the University of Bonn. We would like to thank Katrin S. Reiners from the Institute of Clinical Chemistry and Clinical Pharmacology, University of Bonn for providing the Zetaview NTA. We would like to thank Paula Levermann and Anna Flender for their excellent technical assistance. We acknowledge the measurement of routine laboratory parameters by the Institute of Clinical Chemistry and Clinical Pharmacology and the Institute for Experimental Hematology und Transfusion Medicine (IHT) at the University Hospital Bonn. We would like to thank the participating interventionalists that gathered the blood samples for the study, including Jan Malte Sinning, Marc Ulrich Becher, and Marcel Weber. We thank Dr. Meghan Lucas for her critical reading and valuable suggestions for improvement of the manuscript

Author's contributions Conceptualization, AZ; BAK, FJ, BF, NW; Methodology, AZ; PJ, BAK, AS, VR, JWS, ES, MB; Investigation: PJ, AZ, BAK, VR, MB, MRH, PRG, VT, SZ; Writing - original draft, AZ, Writing-review and editing FJ; BAK, PJ; VR; BF; ES, MB, MRH, PRG, VT, SZ, AS, JWS, NW, GN; Funding acquisition, AZ, FJ, NW, GN; Resources AZ, BAK; MB, MRH, PRG, VT, SZ, AS, JWS, VR, BF, FJ; Supervision FJ, BF, NW, GN.

Funding Open Access funding enabled and organized by Projekt DEAL. This work was supported by the medical faculty of the University of Bonn [BONFOR Grant No. 2018-1A-07 to A.Z., SCIMED Grant: 2018-4-03 to P.J.], by the German Cardiac Society [DGK16/2018 to A.Z.], the Ernst and Berta Grimmke Foundation [13/19 to A.Z.], the Deutsche Forschungsgemeinschaft [WE 4139/8-1 to N.W., JA 2351/2-1 to F.J., and Project-ID 397484323-TRR 259 to F.J.] and the Corona-Foundation [to F.J.].

Data availability Underlying data will be made available by the corresponding authors upon reasonable request.

Conflict of interest The authors report no conflicts of interest.

Ethical approval The study was approved by the ethics committee of the University Hospital of Bonn (283/16).

Consent to participate The study subjects gave written and informed consent to participate.

Consent for publication The study subjects gave written and informed consent to the publication of anonymized data.

Open Access This article is licensed under a Creative Commons Attribution 4.0 International License, which permits use, sharing, adaptation, distribution and reproduction in any medium or format, as long as you give appropriate credit to the original author(s) and the source, provide a link to the Creative Commons licence, and indicate if changes were made. The images or other third party material in this article are included in the article's Creative Commons licence, unless indicated otherwise in a credit line to the material. If material is not included in the article's Creative Commons licence and your intended use is not permitted by statutory regulation or exceeds the permitted use, you will need to obtain permission directly from the copyright holder. To view a copy of this licence, visit http://creativecommons.org/licenses/by/4.0/.

\section{References}

1. Chugh SS, Havmoeller R, Narayanan K, Singh D, Rienstra M, Benjamin EJ et al (2014) Worldwide epidemiology of atrial fibrillation: a Global Burden of Disease 2010 Study. Circulation 129(8):837-847. https://doi.org/10.1161/CIRCULATIONAHA. 113.005119

2. Heeringa J, van der Kuip DA, Hofman A, Kors JA, van Herpen G, Stricker BH et al (2006) Prevalence, incidence and lifetime risk of atrial fibrillation: the Rotterdam study. Eur Heart J 27(8):949-953. https://doi.org/10.1093/eurheartj/ehi825

3. Heijman J, Voigt N, Nattel S, Dobrev D (2014) Cellular and molecular electrophysiology of atrial fibrillation initiation, maintenance, and progression. Circ Res 114(9):1483-1499. https://doi. org/10.1161/CIRCRESAHA.114.302226

4. McAlister FA, Yan L, Roos LL, Lix LM (2019) Parental atrial fibrillation and stroke or atrial fibrillation in young adults. Stroke 50(9):2322-2328. https://doi.org/10.1161/STROKEAHA.119. 025124

5. Palatini P (2004) Parental atrial fibrillation as a risk factor for atrial fibrillation in offspring. JAMA 292(10):1174-1175. https:// doi.org/10.1001/jama.292.10.1174-b

6. Frustaci A, Chimenti C, Bellocci F, Morgante E, Russo MA, Maseri A (1997) Histological substrate of atrial biopsies in patients with lone atrial fibrillation. Circulation 96(4):1180-1184. https://doi.org/10.1161/01.cir.96.4.1180

7. Jahangir A, Lee V, Friedman PA, Trusty JM, Hodge DO, Kopecky SL et al (2007) Long-term progression and outcomes with aging in patients with lone atrial fibrillation: a 30-year follow-up study. Circulation 115(24):3050-3056. https://doi.org/10.1161/CIRCU LATIONAHA.106.644484

8. Cai H, Li Z, Goette A, Mera F, Honeycutt C, Feterik K et al (2002) Downregulation of endocardial nitric oxide synthase expression and nitric oxide production in atrial fibrillation: potential mechanisms for atrial thrombosis and stroke. Circulation 106(22):2854 2858. https://doi.org/10.1161/01.cir.0000039327.11661.16

9. Mawatari K, Yoshioka E, Toda S, Yasui S, Furukawa H, Shimohata $T$ et al (2014) Enhancement of endothelial function inhibits left atrial thrombi development in an animal model of spontaneous left atrial thrombosis. Circ J 78(8):1980-1988. https://doi.org/10. 1253/circj.cj-13-1398

10. Akar JG, Jeske W, Wilber DJ (2008) Acute onset human atrial fibrillation is associated with local cardiac platelet activation and endothelial dysfunction. J Am Coll Cardiol 51(18):1790-1793. https://doi.org/10.1016/j.jacc.2007.11.083

11. Manolio TA, Kronmal RA, Burke GL, O'Leary DH, Price TR (1996) Short-term predictors of incident stroke in older adults. The Cardiovascular Health Study. Stroke 27(9):1479-1486. https://doi.org/10.1161/01.str.27.9.1479

12. Wolf PA, Dawber TR, Thomas HE, Kannel WB (1978) Epidemiologic assessment of chronic atrial fibrillation and risk of stroke: the Framingham study. Neurology 28(10):973-977. https://doi. org/10.1212/wnl.28.10.973 
13. Vanassche T, Lauw MN, Eikelboom JW, Healey JS, Hart RG, Alings $M$ et al (2015) Risk of ischaemic stroke according to pattern of atrial fibrillation: analysis of 6563 aspirin-treated patients in ACTIVE-A and AVERROES. Eur Heart J 36(5):281-287. https://doi.org/10.1093/eurheartj/ehu307

14. Glotzer TV, Daoud EG, Wyse DG, Singer DE, Ezekowitz MD, Hilker C et al (2009) The relationship between daily atrial tachyarrhythmia burden from implantable device diagnostics and stroke risk: the TRENDS study. Circ Arrhythm Electrophysiol 2(5):474-480. https://doi.org/10.1161/CIRCEP.109.849638

15. Kamel H, Okin PM, Elkind MS, Iadecola C (2016) Atrial fibrillation and mechanisms of stroke: time for a new model. Stroke 47(3):895-900. https://doi.org/10.1161/STROKEAHA. 115. 012004

16. Lopez E, Srivastava AK, Burchfield J, Wang YW, Cardenas JC, Togarrati PP et al (2019) Platelet-derived-extracellular vesicles promote Hemostasis and prevent the development of Hemorrhagic shock. Sci Rep 9(1):17676. https://doi.org/10.1038/ s41598-019-53724-y

17. Bucciarelli P, Martinelli I, Artoni A, Passamonti SM, Previtali E, Merati G et al (2012) Circulating microparticles and risk of venous thromboembolism. Thromb Res 129(5):591-597. https:// doi.org/10.1016/j.thromres.2011.08.020

18. Raposo G, Stoorvogel W (2013) Extracellular vesicles: exosomes, microvesicles, and friends. J Cell Biol 200(4):373-383. https:// doi.org/10.1083/jcb.201211138

19. Surgucheva I, Sharov VS, Surguchov A (2012) $\gamma$-Synuclein: seeding of $\alpha$-synuclein aggregation and transmission between cells. Biochemistry 51(23):4743-4754. https://doi.org/10.1021/bi300 478w

20. Hergenreider E, Heydt S, Tréguer K, Boettger T, Horrevoets AJ, Zeiher AM et al (2012) Atheroprotective communication between endothelial cells and smooth muscle cells through miRNAs. Nat Cell Biol 14(3):249-256. https://doi.org/10.1038/ncb2441

21. Choudhury A, Chung I, Blann AD, Lip GYH (2007) Elevated platelet microparticle levels in nonvalvular atrial fibrillation: relationship to p-selectin and antithrombotic therapy. Chest 131(3):809-815. https://doi.org/10.1378/chest.06-2039

22. Azzam H, Zagloul M (2009) Elevated platelet microparticle levels in valvular atrial fibrillation. Hematology 14(6):357-360. https:// doi.org/10.1179/102453309X12473408860460

23. Pourtau L, Sellal JM, Lacroix R, Poncelet P, Bernus O, ClofentSanchez $\mathrm{G}$ et al (2017) Platelet function and microparticle levels in atrial fibrillation: changes during the acute episode. Int J Cardiol 243:216-222. https://doi.org/10.1016/j.ijcard.2017.03.068

24. Mørk M, Andreasen JJ, Rasmussen LH, Lip GYH, Pedersen S, Bæk R et al (2019) Elevated blood plasma levels of tissue factorbearing extracellular vesicles in patients with atrial fibrillation. Thromb Res 173:141-150. https://doi.org/10.1016/j.thromres. 2018.11.026

25. Kirchhof P, Benussi S, Kotecha D, Ahlsson A, Atar D, Casadei B et al (2016) 2016 ESC Guidelines for the management of atrial fibrillation developed in collaboration with EACTS. Eur Heart J 37(38):2893-2962. https://doi.org/10.1093/eurheartj/ehw210

26. Cannon CP, Brindis RG, Chaitman BR, Cohen DJ, Cross JT, Drozda JP et al (2013) 2013 ACCF/AHA key data elements and definitions for measuring the clinical management and outcomes of patients with acute coronary syndromes and coronary artery disease: a report of the American College of Cardiology Foundation/American Heart Association Task Force on Clinical Data Standards (Writing Committee to Develop Acute Coronary Syndromes and Coronary Artery Disease Clinical Data Standards). Crit Pathw Cardiol 12(2):65-105. https://doi.org/10.1097/HPC. 0b013e3182846e16

27. Baumgartner H, Hung J, Bermejo J, Chambers JB, Edvardsen T, Goldstein S et al (2017) Recommendations on the echocardiographic assessment of aortic valve stenosis: a focused update from the European Association of Cardiovascular Imaging and the American Society of Echocardiography. J Am Soc Echocardiogr 30(4):372-392. https://doi.org/10.1016/j.echo.2017. 02.009

28. Lancellotti P, Tribouilloy C, Hagendorff A, Popescu BA, Edvardsen T, Pierard LA et al (2013) Recommendations for the echocardiographic assessment of native valvular regurgitation: an executive summary from the European Association of Cardiovascular Imaging. Eur Heart J Cardiovasc Imaging 14(7):611-644. https:// doi.org/10.1093/ehjci/jet105

29. Baumgartner H, Hung J, Bermejo J, Chambers JB, Evangelista A, Griffin BP et al (2009) Echocardiographic assessment of valve stenosis: EAE/ASE recommendations for clinical practice. J Am Soc Echocardiogr 22(1):1-23. https://doi.org/10.1016/j. echo.2008.11.029 (quiz 101-2)

30. Jansen F, Yang X, Hoelscher M, Cattelan A, Schmitz T, Proebsting $S$ et al (2013) Endothelial microparticle-mediated transfer of MicroRNA-126 promotes vascular endothelial cell repair via SPRED1 and is abrogated in glucose-damaged endothelial microparticles. Circulation 128(18):2026-2038. https://doi.org/ 10.1161/CIRCULATIONAHA.113.001720

31. Liu Y, Li Q, Hosen MR, Zietzer A, Flender A, Levermann P et al (2018) Atherosclerotic conditions promote the packaging of functional MicroRNA-92a-3p into Endothelial microvesicles. Circ Res. https://doi.org/10.1161/CIRCRESAHA.118.314010

32. Rolfes V, Ribeiro LS, Hawwari I, Böttcher L, Rosero N, Maasewerd S et al (2020) Platelets fuel the inflammasome activation of innate immune cells. Cell Rep 31(6):107615. https:// doi.org/10.1016/j.celrep.2020.107615

33. Gray WD, Mitchell AJ, Searles CD (2015) An accurate, precise method for general labeling of extracellular vesicles. MethodsX 2:360-367. https://doi.org/10.1016/j.mex.2015.08.002

34. van der Pol E, van Gemert MJ, Sturk A, Nieuwland R, van Leeuwen TG. Single vs. swarm detection of microparticles and exosomes by flow cytometry. J Thromb Haemost. 2012;10(5):919-30. https://doi.org/10.1111/j.1538-7836.2012. 04683.x.

35. Parida BK, Garrastazu H, Aden JK, Cap AP, McFaul SJ (2015) Silica microspheres are superior to polystyrene for microvesicle analysis by flow cytometry. Thromb Res 135(5):1000-1006. https://doi.org/10.1016/j.thromres.2015.02.011

36. Zietzer A, Steffen E, Niepmann S, Düsing P, Hosen MR, Liu W et al (2020) MicroRNA-mediated vascular intercellular communication is altered in chronic kidney disease. Cardiovasc Res. https:// doi.org/10.1093/cvr/cvaa322

37. Wilfinger WW, Mackey K, Chomczynski P. Effect of $\mathrm{pH}$ and ionic strength on the spectrophotometric assessment of nucleic acid purity. Biotechniques. 1997;22(3):474-6, 8-81. https://doi.org/ 10.2144/97223st01.

38. Thermo Fisher Scientific: interpretation of Nucleic Acid 260/280 Ratios. https://assets.fishersci.com/TFS-Assets/CAD/ProductBulletins/T123-NanoDrop-Lite-Interpretation-of-Nucleic-Acid260-280-Ratios.pdf (2012). Accessed 12/02/2020.

39. Zietzer A, Hosen MR, Wang H, Goody PR, Sylvester M, Latz E et al (2020) The RNA-binding protein hnRNPU regulates the sorting of microRNA-30c-5p into large extracellular vesicles. J Extracell Vesicles 9(1):1786967. https://doi.org/10.1080/20013 078.2020.1786967

40. Ambrose AR, Alsahli MA, Kurmani SA, Goodall AH (2018) Comparison of the release of microRNAs and extracellular vesicles from platelets in response to different agonists. Platelets 29(5):446-454. https://doi.org/10.1080/09537104.2017.1332366

41. Zapilko V, Fish RJ, Garcia A, Reny JL, Dunoyer-Geindre S, Lecompte T et al (2020) MicroRNA-126 is a regulator of 
platelet-supported thrombin generation. Platelets 31(6):746-755. https://doi.org/10.1080/09537104.2020.1775804

42. Alexandru N, Constantin A, Nemecz M, Comariţa IK, Vîlcu A, Procopciuc A et al (2019) Hypertension associated with hyperlipidemia induced different MicroRNA expression profiles in plasma, platelets, and platelet-derived microvesicles; effects of endothelial progenitor cell therapy. Front Med (Lausanne) 6:280. https://doi. org/10.3389/fmed.2019.00280

43. Edelstein LC, McKenzie SE, Shaw C, Holinstat MA, Kunapuli SP, Bray PF (2013) MicroRNAs in platelet production and activation. J Thromb Haemost 11(Suppl 1):340-350. https://doi.org/10.1111/ jth.12214

44. Jansen F, Wang H, Przybilla D, Franklin BS, Dolf A, Pfeifer P et al (2016) Vascular endothelial microparticles-incorporated microRNAs are altered in patients with diabetes mellitus. Cardiovasc Diabetol 15:49. https://doi.org/10.1186/s12933-016-0367-8

45. Geddings JE, Hisada Y, Boulaftali Y, Getz TM, Whelihan M, Fuentes R et al (2016) Tissue factor-positive tumor microvesicles activate platelets and enhance thrombosis in mice. J Thromb Haemost 14(1):153-166. https://doi.org/10.1111/jth.13181

46. Lima LG, Oliveira AS, Campos LC, Bonamino M, Chammas R, Werneck C et al (2011) Malignant transformation in melanocytes is associated with increased production of procoagulant microvesicles. Thromb Haemost 106(4):712-723. https://doi.org/10.1160/ TH11-03-0143
47. Campello E, Spiezia L, Radu CM, Bulato C, Castelli M, Gavasso $S$ et al (2011) Endothelial, platelet, and tissue factor-bearing microparticles in cancer patients with and without venous thromboembolism. Thromb Res 127(5):473-477. https://doi.org/10. 1016/j.thromres.2011.01.002

48. Jansen F, Stumpf T, Proebsting S, Franklin BS, Wenzel D, Pfeifer $P$ et al (2017) Intercellular transfer of miR-126-3p by endothelial microparticles reduces vascular smooth muscle cell proliferation and limits neointima formation by inhibiting LRP6. J Mol Cell Cardiol 104:43-52. https://doi.org/10.1016/j.yjmcc.2016.12.005

49. Poliseno L, Tuccoli A, Mariani L, Evangelista M, Citti L, Woods $\mathrm{K}$ et al (2006) MicroRNAs modulate the angiogenic properties of HUVECs. Blood 108(9):3068-3071. https://doi.org/10.1182/ blood-2006-01-012369

50. Shi L, Fisslthaler B, Zippel N, Frömel T, Hu J, Elgheznawy A et al (2013) MicroRNA-223 antagonizes angiogenesis by targeting $\beta 1$ integrin and preventing growth factor signaling in endothelial cells. Circ Res 113(12):1320-1330. https://doi.org/10.1161/ CIRCRESAHA.113.301824

51. Laffont B, Corduan A, Plé H, Duchez AC, Cloutier N, Boilard E et al (2013) Activated platelets can deliver mRNA regulatory Ago2 $\bullet$ microRNA complexes to endothelial cells via microparticles. Blood 122(2):253-261. https://doi.org/10.1182/ blood-2013-03-492801 